\title{
Dexamethasone in the management of symptoms due to herniated lumbar disc ${ }^{1}$
}

\author{
L. N. GREEN
}

From the division of Neurology, Department of Medicine, Youngstown Hospital Association, Gypsy Lane and Goleta, Youngstown, Ohio 44505, U.S.A.

SYNOPSIS One hundred consecutive patients with radicular pain due to myelographically proven herniated lumbar intervertebral discs were treated with initially high but tapering doses of intramuscular dexamethasone for seven days. All patients had relief of pain within 24-48 hours. Bed rest was eliminated as a significant factor in the improvement. Nine patients required surgery at the end of the hospital treatment period. Another 11 required surgery during the follow-up of 15 months. Review of work status and recurrent pain during the follow-up indicates that the non-surgically treated patients in this series fared better. It is concluded that nerve root inflammation is the immediate cause of radicular symptoms in case of ruptured lumbar disc and that treatment with dexamethasone gives prompt relief of pain and may avoid the need for surgery in most cases.

It is well known that there are a variety of methods for treating symptoms related to lumbar radiculopathy caused by a herniated or prolapsed intervertebral disc. Traditionally, these methods have employed so called 'conservative' (non-surgical) or surgical techniques, each having its own group of advocates and practitioners. Surgical treatment, besides having an inherent risk of surgical and anaesthetic mortality, bears many other hazards. These include paralysis of the foot (Youmans, 1973), disturbing paraesthesias, adhesive arachnoiditis with resultant paraplegia and bladder dysfunction (Smolik and Nash, 1951), wound infection, and thrombophlebitis (Raaf, 1959), as well as persistence and even aggravation of the original pain for which operation was initially undertaken (Spurling and Grantham, 1949; Smolik and Nash, 1951; Raaf, 1959; Rosen, 1969). Conservative therapy, on the other hand, frequently requires prolonged bed rest and may be associated with protracted disability (Friedenberg and Shoemaker, 1954; Millikan, 1962). More recently, intradiscal injection of chymopapain has come into vogue, but this requires a degree of

\footnotetext{
${ }^{1}$ Presented, in part, at the 26th Annual Meeting of the American Academy of Neurology, San Francisco, Cal., 1974.

(Accepted 27 June 1975)
}

skill and special training and also bears specific risks (Wiltse et al., 1975). Consequently, a treatment which could rapidly alleviate the symptoms and signs due to herniated lumbar disc, and not require the mastery of a new technique, would be a valuable addition to our therapeutic armamentarium.

The use of corticosteroids for the relief of disc symptoms (Feffer, 1956; Naylor and Turner, 1961; Segal and Gardner, 1962; Dilke et al., 1973) has been based upon the observation that inflammatory and oedematous changes in and around the nerve root are present (Irsigler, 1951; Lindahl and Rexed, 1951; Berg, 1953; Rosen, 1969; Marshall and Trethewie, 1973) and may play a major role in the development of those symptoms. In order to assess the possible benefit that might occur from the use of corticosteroids 100 consecutive patients with myelographically proven disc herniations were treated with a short course of high doses of intramuscularly administered dexamethasone in this study.

\section{METHOD}

One hundred consecutive patients were hospitalized because of radicular pain of lumbar origin and 
were evaluated clinically and myelographically. The duration of symptoms varied from four days to many months. There were 43 women and 57 men whose ages ranged from 17 to 87 years, the average being 51.4 years. Sixty-seven per cent of the patients were between 40 and 69 years old. Their occupations varied from sedentary to hard labour. Twenty-one patients were retired before entering the study. Eighty-five patients had a positive Lasègue sign. Fifty had limitation of lumbosacral spinal motion, and pain was elicited or aggravated by these motions. Fifty-one had weakness of the dorsal toe extensor and anterior tibialis muscles and 46 had depression or absence of deep tendon reflexes. All patients had myelographic defects diagnostic of root compression at a lumbar disc space. Fourteen patients had multiple defects. The disc spaces involved were: L1-L2, one instance; L2-L3, three instances; L3L4, 21 instances; L4-L5, 67 instances; L5-S1, 27 instances. Six patients had diabetes mellitus.

All patients were treated with daily intramuscular injections of dexamethasone in divided dosage according to the following schedule: first day, 64 $\mathrm{mg}$; second day, $32 \mathrm{mg}$; third day, $24 \mathrm{mg}$; fourth day, $12 \mathrm{mg}$; fifth through the seventh day, $8 \mathrm{mg}$ per day. Before treatment with dexamethasone 15 patients had been treated with bed rest alone for an average of 13.3 days (range seven to 21 days) without satisfactory relief of pain. Twenty patients were allowed to walk freely during the week of steroid treatment. Eighty patients were restricted to bed (except for bathroom privileges) during the course of treatment. On the eighth day after beginning treatment the dexamethasone was discontinued in all patients, and those who were restricted to bed rest were allowed to walk. On the ninth day, on the basis of the patient's response, a decision was made whether the patient could be discharged and followed as an out-patient or if surgery were indicated.

Long term follow-up was obtained by outpatient examination and by questionnaire. The duration of follow-up from the time of initial hospitalization varied between three and 34 months, the average being 15.4 months for the entire group of 100 patients. At no time during the follow-up period was dexamethasone given again. The questionnaire was answered by the patient at the end of the follow-up period and was designed to give his or her assessment of residual symptoms and limitations. In regard to residual pain the patient was asked to specify which of the following best described his or her condition:

A. I have been completely free of pain

B. I have mild pain once in a while (rarely)

C. I have mild pain frequently

D. I have mild pain constantly
E. I have severe pain once in a while (rarely)

F. I have severe pain frequently

G. I have severe pain constantly

The above pain categories are in increasing order of severity. The questionnaire also determined whether patients were able to return to previous employment, the type of work, and if disc surgery had been performed in the follow-up period and the results thereof.

There was no significant difference between the group of patients ultimately requesting surgery and the non-surgical category in regard to the degree of physical labour involved in their occupations.

\section{RESULTS}

During the initial hours of treatment all patients noted marked decrease in pain. This was manifest not only in their subjective response but also in the fact that those for whom pain medication had been previously ordered stopped asking for it. Also considered to be a subjective sign of improvement was their expressed desire to walk even when pain had previously restricted them to bed rest, in some cases for as much as three weeks before treatment. Despite the marked relief or complete absence of pain, the first 80 patients in the series were urged to remain at bed rest. The next 20 patients in the series were allowed free ambulation. At the end of the treatment period 10 patients who noted return of pain expressed a desire for surgery, although they readily admitted that their pain was less severe than before treatment. Two of these were refused surgery at that time because of multiple level involvement. Eight had surgery. One additional patient had discectomy because of persistent profound foot drop, even though he was completely free of pain. The foot drop did not improve after the operation. Consequently, 90 patients were sufficiently free of pain during the initial course of treatment to be discharged from hospital nine days after beginning treatment. Nine patients had surgery during the initial hospitalization. None of the patients who had been freely ambulatory during the course of dexamethasone treatment required surgery during the index hospitalization.

In the first 24-48 hours of treatment the mechanical signs of herniated lumbar discnamely, a positive Lasègue sign and restriction of lumbar spinal motion-improved coincidently 


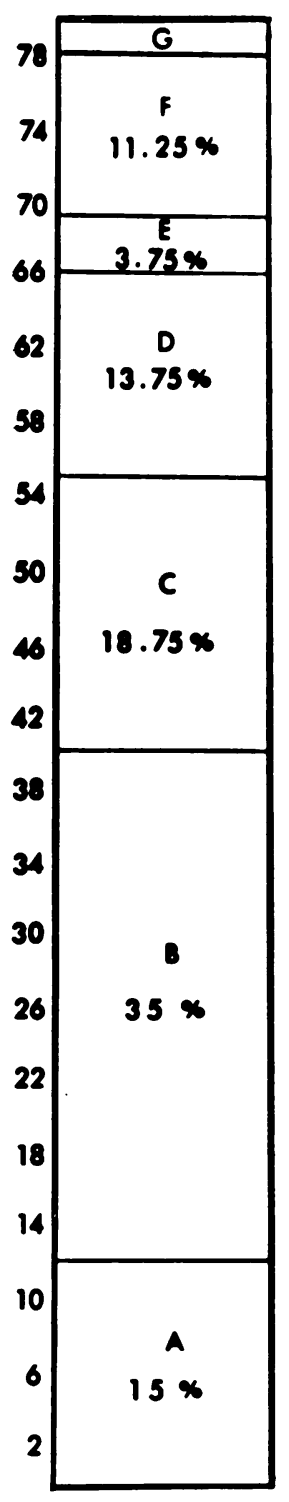

NON SURGICAL

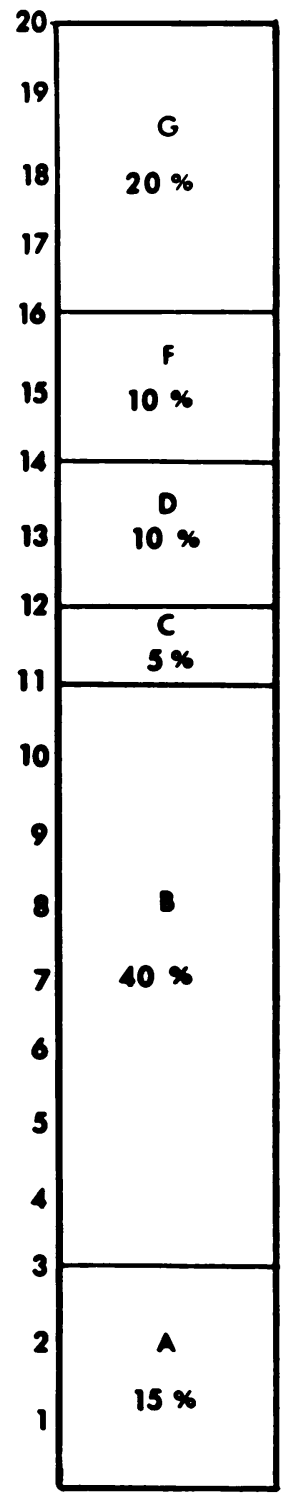

SURGICAL

FIG. 1 Comparison of residual pain at long-term follow-up. The height of each column indicates number of patients within each pain category, in proportion to the total. A. No pain. B. Mild pain rarely. C. Mild pain frequently. D. Mild pain constantly. E. Severe pain rarely. F. Severe pain frequently. G. Severe pain constantly.

with the relief of pain. In 68 of 85 cases who had a positive Lasègue sign and in 31 of 50 cases with limited spinal movement there was either complete absence of these signs or marked improvement within the first 48 hours. Mild to moderate weakness of leg or foot muscles improved or disappeared in 40 of 51 cases in which it was found. In no case where there was loss of a reflex was it noted to return, although mildly depressed reflexes did return to normal. Also, areas of hypalgesia returned to normal sensation.

No complications of steroid therapy were noted during the seven days of treatment. However, one woman developed a deep calf thrombophlebitis a few days after discharge from hospital which subsequently subsided without further untoward consequences.

With regard to the symptom of pain during the follow-up period, return of pain was severe enough in 11 additional patients to warrant surgery for disc removal. Eighty $(80 \%)$ patients did not have surgery during the follow-up period nor during the initial hospitalization and their assessment of pain symptoms during this period is as follows (Fig. 1): there were 12 responses of 'completely free of pain', 28 responses of 'mild pain once in a while (rarely)', 15 responses of 'mild pain frequently', 11 responses of 'mild pain constantly', three responses of 'severe pain once in a while (rarely)', nine responses of 'severe pain frequently', and two responses of 'severe pain constantly'. Thus, during the follow-up period in the 80 patients who did not have surgery, or any other specific treatment, the incidence of recurrent mild pain was $67.5 \%$, and in $15 \%$ there was no recurrence of pain. In a total of $82.5 \%$ of the responses to the query regarding pain there was either no pain or only mild pain. The 20 patients who had discectomy reported the following response to the pain question upon long-term follow-up (Fig. 1): four had 'severe pain constantly' two had 'severe pain frequently', two had 'mild pain constantly', one had 'mild pain frequently', eight had 'mild pain once in a while (rarely)', and three were 'completely free of pain'. Thus on follow-up of the surgical group, $55 \%$ had recurrence of mild pain and $15 \%$ had no pain. Therefore, $30 \%$ of the surgical group had recurrence of severe pain, while $70 \%$ were either pain free or had only mild pain. The comparable 
TABLE

WORK STATUS IN 80 NON-SURGICAL AND 20 POST-SURGICAL DISC PATIENTS AT TIME OF FOLLOW-UP

\begin{tabular}{|c|c|c|c|c|c|}
\hline & Retired & Disabled & $\begin{array}{c}\text { Lesser } \\
\text { job }\end{array}$ & Same job & Total \\
\hline $\begin{array}{l}\text { Surgery } \\
\text { No } \\
\text { surgery }\end{array}$ & $3(15 \%)$ & $6(30 \%)$ & $3(15 \%)$ & $8(40 \%)$ & 20 \\
\hline
\end{tabular}

Actual numbers and percentage of patients within each group are stated. The retired group had been retired before entry into the study.

figures for the non-surgical group would be $17.5 \%$ and $82.5 \%$ (Fig. 1). One patient who had surgery and suffered bowel and bladder complications in addition to aggravation of pain committed suicide 11 months after surgery. Of the 80 non-surgically treated patients, 54 were able to return to their former occupations, one had to accept a less physically active job, seven were completely disabled, and 18 were retired before entering the study. In the surgical group, the comparable figures were eight returned to former job, three took less strenuous job, six were completely disabled, and three had retired before entering the study (Table).

\section{DISCUSSION}

Since the original clinical description by Mixter and Barr (1934), the belief has been held that the symptoms and signs produced by a herniated lumbar disc are due to mechanical compression of the nerve root by the disc material (Charlton, 1962; Crock, 1970). Little attention has been paid to the observation that the nerve roots may appear swollen, hyperaemic, and inflamed when exposed at surgery (Raaf, 1959). Swollen roots can also be seen myelographically (Berg, 1953). The fact that between $35 \%$ and $63 \%$ of patients with ruptured lumbar discs have an elevation of spinal fluid protein (Albert et al., 1955; Raaf, 1959; Rosen, 1969) would also be in favour of an inflammatory process in the pathogenesis of symptoms and signs. In a direct histological study of biopsy specimens from the posterior nerve root exposed at operation in patients with sciatica, Lindahl and Rexed (1951) found inflammatory changes in seven out of 10 cases. Irsigler (1951), in a similar study, found acute or chronic inflammatory changes in the nerve roots of patients who had prolapsed lumbar discs. Berg, in a myelographic study in 1953 noted the reduction in the size of swollen lumbar roots coincident with clinical improvement in the absence of any change in the myelographic appearance of the disc protrusion. He stated, 'It is not the disc protrusion as such but secondary pathoanatomic changes which are responsible for the symptoms. These changes are probably inflammatory in nature, taking the

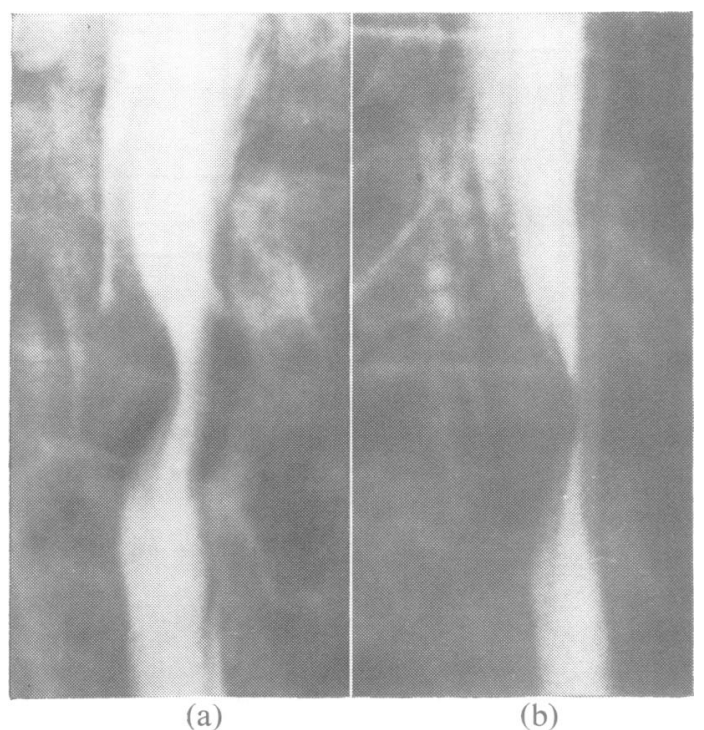

FIG. 2 Close-up of oblique views of myelogram taken at 40 inch tube to target distance in 33 year old man showing herniated disc at L4-L5 interspace. (a) Before treatment. (b) Six days after beginning dexamethasone. The decrease in nerve root swelling after treatment is readily apparent.

appearance of edema of the roots'. A reduction in nerve root swelling occurred while a 33 year old patient in the current study was on dexamethasone therapy, without change in the size of the herniated disc (Fig. 2). There are other studies that demonstrate that ruptured lumbar disc and nerve root compression may coexist without symptoms (Hitselberger and Witten, 1968) and that patients with herniated discs may have complete remission after conservative treatment without a change in the myelographic 
appearance of the disc (Falconer et al., 1949). These findings favour the view that mechanical compression alone is not a sufficient cause of the symptoms seen in ruptured disc disease.

The logical therapeutic extension of the concept that an inflammatory process plays a major role in the morbidity of ruptured lumbar discs is the use of corticosteroids. Jones and Barnett (1955) reported that when they suffused the epidural space with 25 to $50 \mathrm{mg}$ hydrocortisone during surgery for removal of a ruptured disc their patients had a much less painful postoperative course than in similarly operated patients who were not so treated. Segal and Gardner (1962) reported encouraging results with the use of intrathecally administered corticosteroids. In the process of performing discograms, Feffer (1956) injected $50 \mathrm{mg}$ hydrocortisone into the ruptured disc of his patients suffering from sciatica. He noted that symptoms remitted rapidly in $67 \%$ of these patients. Five of his patients who had normal discograms showed no improvement. A 48 hour intravenous infusion of ACTH produced either moderate or complete relief of symptoms and signs in 24 of 38 patients thought to be suffering from lumbar disc prolapse by Naylor and Turner (1961). The relief became apparent within 24 hours of infusion and was well developed at 48 hours. More recently, Dilke and co-authors (1973) reported on the use of $80 \mathrm{mg}$ methylprednisolone injected extradurally in a controlled double-blind trial of 100 consecutive patients who were diagnosed as having root compression by a herniated disc. They found that the relief of pain was significantly greater and more frequent in the treated than in the non-treated group. They attributed improvement to the anti-inflammatory properties of the steroid. However, it should be noted that their patients were not studied myelographically before randomization.

In this study, 100 consecutive patients with myelographically proven herniated discs who had signs and symptoms were treated in a standardized fashion with intramuscular dexamethasone, initially with high but tapering dosage for a period of seven days. An example of the effect of this treatment on the reduction of nerve root swelling is seen in Fig. 2. The rapid improvement in signs and symptoms in a period of 24-48 hours is similar to other reports in the literature in which other preparations of corticosteroid medication were administered by other routes (Feffer, 1956; Naylor and Turner, 1961).

Although bed rest was prescribed coincidentally with the dexamethasone in the majority of patients, it is highly unlikely that such rapid improvement could have occurred with bed rest alone. Fifteen of the patients in this series had between seven and 21 days (average 13.3 days) of complete bed rest without satisfactory improvement of pain until treatment with dexamethasone started. Within 24-48 hours of beginning treatment these patients, as well as all others on this regimen, showed rapid relief or disappearance of pain. This occurred even in the 20 patients who had been allowed to walk about freely, demonstrating that bed rest was not essential to the reversal of symptoms and signs. Pearce and Moll (1967), in a study of patients with acute lumbar disc lesions treated by bed rest alone, found that a minimum of two weeks, and more often three weeks, were required for very gradual relief of pain. Their patients were hospitalized for a minimum of three to five weeks. In a study of 36 conservatively treated patients with myelographically proven lumbar disc herniations, Friedenberg and Shoemaker (1954) found that a minimum of one to three weeks of bed rest was required for improvement of pain and that at the end of this period the patients needed a back support to sustain relief. Although it appears likely that restriction to bed is of benefit in reducing symptoms of disc herniation, the relief of pain afforded by bed rest alone is a gradual slow process requiring several weeks in most cases. By contrast, the consistent improvement in the 20 ambulatory patients in this study, as well as the rapid improvement after starting dexamethasone in the 15 patients who had not previously responded satisfactorily to bed rest alone, would tend to mitigate the role of bed rest as compared with dexamethasone therapy.

Towards the end of the treatment period, when the dose of dexamethasone was lowest, some patients noted increasing amounts of pain, but never as severe as before treatment began. When some of these patients were treated again with the initial high dose of dexamethasone their pain disappeared.

Of the 10 patients in this study who were not 
satisfied with the degree of pain relief at the end of their hospital treatment, eight had surgery. It should be noted that these patients were no different from the remaining 92 in their initial pain relief. It did not appear that consideration of age entered into the decision for surgery, since these cases varied from 17 to 73 years old. Neither did it seem that consideration of the strenuousness of occupation was a major factor, since these patients rated their occupations as no more physically taxing than did the group who did not have surgery. Some retired patients opted for surgery, whereas several labourers were in the non-surgical group. One patient who had surgery during the initial hospitalization was satisfied with the degree of pain relief but surgery was recommended because of persistent foot drop which, however, did not improve in the postoperative follow-up. In this regard, Anderson and Carlsson (1966) observed that only $47 \%$ of patients who had surgery for lumbar disc herniation associated with foot extensor paralysis showed some improvement in the paralysis. They concluded that foot extensor paralysis need not be considered an indication for discectomy and observed that relief from pain was the most important factor in choosing treatment.

The long term natural history of non-surgically treated patients with herniated lumbar discs is not well known. There are few studies in the literature which discuss this subject. During a long-term follow-up averaging eight years in 68 patients, Pearce and Moll (1967) found that $7 \%$ required surgery for relief of symptoms. This compares with a surgical rate of 11 out of 91 subjects $(12 \%)$ who needed surgery during the average follow-up period of slightly over 15 months in the current study. It would be expected that the non-surgically treated patients in this study should follow the natural history of lumbar disc disease and be comparable with the study of Pearce and Moll. In the 80 nonsurgically treated patients, $15 \%$ indicated absence of pain on long term follow-up, whereas $67.5 \%$ reported mild pain, and $17.5 \%$ noted severe pain. This compares with $26.5 \%$ who reported no pain, $41 \%$ who had mild pain, and $32.4 \%$ who had severe pain in the series of Pearce and Moll (1967). It should be noted that their cases were not myelographically verified.
After an average follow-up of five years, Friedenberg and Shoemaker (1954) found that $47 \%$ of 36 patients with myelographically proven discs were pain free, whereas $31 \%$ had mild residual pain and $22 \%$ had severe residual pain. From these figures it would appear reasonable to predict that, after an acute attack of pain due to herniated lumbar disc disease has subsided, the large majority of patients will have either no further recurrence or only mild residual pain.

Of great significance to the individual patient is his or her ability to resume gainful employment after being disabled by disc symptoms. In the long-term follow-up in this study, the chances of not being able to return to the same employment, either because of complete or partial disability, were $45 \%$ in the surgical group as compared with $10 \%$ in the non-surgical group. The chances of being able to return to the same job as before the illness was greater in the non-surgical group $(67.5 \%)$ than in the surgically treated group $(40 \%)$ (Table). These differences were not significantly affected when the number of retired people were discounted in each group. From the data it would appear that the patients who did not have surgery fared considerably better in regard to employment than those who underwent discectomy.

It would be interesting to know if a repeated brief course of treatment with dexamethasone sometime during the long-term follow-up period would have been of benefit in some of the patients who had a recurrence of severe pain, and whether such treatment could have avoided surgery in the 11 patients who had discectomy during that period. This question is currently being evaluated.

This study indicates that the use of the antiinflammatory agent, dexamethasone, is a valuable addition to the current therapeutic armamentarium for relieving the symptoms and signs of acute lumbar disc herniation. It is easily administered, has a rapid onset of action, and appears to be uniformly effective without serious side-effects when used in the manner reported. While this study cannot be considered a valid comparison with the results of surgical treatment (because of the small number of surgical cases) it offers hope that more patients with a lumbar disc lesion can be treated satisfactorily without surgery. 


\section{REFERENCES}

Albert, S. M., Rechtman, A. M., and Kremens, V. (1955). Significance of spinal fluid protein levels in disc pathology. Pennsylvania Medicine, 58, 1235-1236.

Anderson, H., and Carlsson, C. (1966). Prognosis of operatively treated lumbar disc herniations causing foot extensor paralysis. Acta Chirurgica Scandinavica, 132, 501506.

Berg, A. (1953). Clinical and myelographic studies of conservatively treated cases of lumbar intervertebral disc protrusion. Acta Chirurgica Scandinavica, 104, 124-129.

Charlton, W. S. (1962). Review of the present position in spinal intervertebral disc disease. Medical Journal of Australia, 49, 581-586.

Crock, H. V. (1970). A reappraisal of intervertebral disc lesions. Medical Journal of Australia, 1, 983-989.

Dilke, T. F. W., Bury, H. C. W., and Grahame, R. (1973). Extradural corticosteroid injection in management of lumbar nerve root compression. British Medical Journal, 2, 635-637.

Falconer, M., McGeorge, M., and Begg, A. (1949). Observations of the cause and mechanism of symptom production in sciatica and low back pain. Journal of Neurology, Neurosurgery, and Psychiatry, 11, 13-15.

Feffer, H. L. (1956). Treatment of low back and sciatic pain by injection of hydrocortisone into degenerated discs. Journal of Bone and Joint Surgery, 38-A, 585-592.

Friedenberg, L. B., and Shoemaker, R. C. (1954). Results of non-operative treatment of ruptured lumbar discs. American Journal of Surgery, 88, 933-935.

Hitselberger, W. E., and Witten, R. M. (1968). Abnormal myelograms in asymptomatic patients. Journal of Neurosurgery, 28, 204-206.

Irsigler, F. J. (1951). Microscopic findings in spinal cord roots of patients with lumbar and lumbosacral disc prolapse. Acta Neurochirurgica, 1, 478-516.

Jones, K. G., and Barnett, H. C. (1955). Use of hydrocorti- sone in spinal surgery. Southern Medical Journal, 48, 617623.

Lindahl, D., and Rexed, B. (1951). Histological changes in spinal nerve roots of operated cases of sciatica. Acta Orthopedica Scandinavica, 20, 215-225.

Marshall, L. L., and Trethewie, E. R. (1973). Chemical irritation of nerve root in disc prolapse. Lancet, 2, 320.

Millikan, C. H. (1962). Problems of evaluating treatment of protruded lumbar invertebral disk: observations of results of conservative and surgical treatment in 429 cases. Journal of American Medical Association, 155, 291-293.

Mixter, W. J., and Barr, J. S. (1934). Rupture of the intervertebral disc with involvement of the spinal canal. New England Journal of Medicine, 211, 210-215.

Naylor, A., and Turner, R. L. (1961). ACTH in the treatment of lumbar disc prolapse. Proceedings of the Roya Society of Medicine, 54, 282-284.

Pearce, J., and Moll, J. M. (1967). Conservative treatment and natural history of acute lumbar disc lesions. Journal of Neurology, Neurosurgery, and Psychiatry, 30, 13-17.

Raaf, J. (1959). Some observations regarding 905 patients operated upon for protruded lumbar intervertebral disc. American Journal of Surgery, 97, 388-397.

Rosen, H. J. (1969). Lumbar intervertebral disc surgery: review of 300 cases. Canadian Medical Association Journal, 101, 317-323.

Segal, A. D., and Gardner, W. J. (1962). Subarachnoid injection with corticosteroids in management of sciatica. Journal of Indian Medical Association, 30, 291-293.

Smolik, E. A., and Nash, F. O. (1951). Lumbar spinal arachnoiditis: complication of intervertebral disc operation. Annals of Surgery, 133, 490-495.

Spurling, R. G., and Grantham, E. G. (1949). End results of surgery for ruptured lumbar intervertebral discs: follow up study of 327 cases. Journal of Neurosurgery, 6, 57-64.

Wiltse, L. L., Widell, E. H. (Jr), and Hansen, A. Y. (1975). Chymopapain chemonucleolysis in lumbar disk disease. Journal of the American Medical Association, 231, 474-479.

Youmans, J. R. (1973) (ed.). Neurological Surgery, vol. 2, p. 1176. Saunders: London. 